\title{
A More Accurate Half-Discrete Hilbert Inequality with a Nonhomogeneous Kernel
}

\author{
Qiliang Huang and Bicheng Yang \\ Department of Mathematics, Guangdong University of Education, Guangzhou, Guangdong 510303, China \\ Correspondence should be addressed to Bicheng Yang; bcyang@gdei.edu.cn
}

Received 8 May 2013; Accepted 3 June 2013

Academic Editor: Kehe Zhu

Copyright (C) 2013 Q. Huang and B. Yang. This is an open access article distributed under the Creative Commons Attribution License, which permits unrestricted use, distribution, and reproduction in any medium, provided the original work is properly cited.

By using the way of weight functions and the idea of introducing parameters, a more accurate half-discrete Hilbert inequality with a nonhomogeneous kernel and a best constant factor is presented. We also consider its best extension with the parameters, the equivalent forms, and the operator expressions as well as some reverses.

\section{Introduction}

If $a_{n}, b_{n} \geq 0, a=\left\{a_{n}\right\}_{n=1}^{\infty} \in l^{2}, b=\left\{b_{n}\right\}_{n=1}^{\infty} \in l^{2}$, and $\|a\|=$ $\left\{\sum_{n=1}^{\infty} a_{n}^{2}\right\}^{1 / 2}>0$ and $\|b\|=\left\{\sum_{n=1}^{\infty} b_{n}^{2}\right\}^{1 / 2}>0$, then we have the following discrete Hilbert inequality (cf. [1]):

$$
\sum_{n=1}^{\infty} \sum_{m=1}^{\infty} \frac{a_{m} b_{n}}{m+n}<\pi\|a\|\|b\|
$$

where the constant factor $\pi$ is the best possible. Moreover, for $f, g(\geq 0) \in L^{2}\left(R_{+}\right),\|f\|=\left\{\int_{0}^{\infty} f^{2}(x) d x\right\}^{1 / 2}>0$, and $\|g\|>0$, we have the following Hilbert integral inequality (cf. $[2]):$

$$
\iint_{0}^{\infty} \frac{f(x) g(y)}{x+y} d x d y<\pi\|f\|\|g\|,
$$

with the same best constant factor $\pi$. Inequalities (1)-(2) are important in the analysis and its applications (cf. [3]). There are lots of improvements, generalizations, and applications of inequalities (1)-(2) for more details, refer to the literatures [4$15]$.

We find a result on the half-discrete Hilbert-type inequality with the nonhomogeneous kernel, which were published early as follows (cf. [16, Theorem 351]):

$$
\int_{0}^{\infty} x^{q-2}\left(\sum_{n=1}^{\infty} \frac{a_{n}}{1+x n}\right)^{q} d x<B^{q}\left(\frac{1}{p}, \frac{1}{q}\right) \sum_{n=1}^{\infty} a_{n}^{q},
$$

where $p>1, q>1,1 / p+1 / q=1$, and $B(u, v)=$ $\int_{0}^{\infty}\left(t^{u-1} d t /(1+t)^{u+v}\right)(u, v>0)$ is the beta function. But the constant factors $B(1 / p, 1 / q)$ were not considered whether the best possible. Recently, Yang gave a half-discrete Hilbert inequality with the nonhomogeneous kernel as follows (cf. [17]):

$$
\sum_{n=1}^{\infty} a_{n} \int_{0}^{\infty} \frac{f(x)}{1+x n} d x<\pi\left(\sum_{n=1}^{\infty} a_{n}^{2} \int_{0}^{\infty} f^{2}(x) d x\right)^{1 / 2},
$$

where the constant factor $\pi$ is the best possible. And some half-discrete Hilbert-type inequalities with the nonhomogeneous kernel were given (cf. [18]).

In this paper, by using the way of weight functions and the idea of introducing parameters and by means of Hadamard's inequality, we give the more accurate inequalities of (3) and (4) as follows:

$$
\begin{aligned}
& \int_{0}^{\infty} x^{q-2}\left(\sum_{n=1}^{\infty} \frac{a_{n}}{1+x n-x / 2}\right)^{q} d x<B^{q}\left(\frac{1}{p}, \frac{1}{q}\right) \sum_{n=1}^{\infty} a_{n}^{q}, \\
& \sum_{n=1}^{\infty} a_{n} \int_{0}^{\infty} \frac{f(x)}{1+x n-x / 2} d x<\pi\left(\sum_{n=1}^{\infty} a_{n}^{2} \int_{0}^{\infty} f^{2}(x) d x\right)^{1 / 2},
\end{aligned}
$$

with the best constant factors $B(1 / p, 1 / q)$ and $\pi$. We also consider their best extensions with the parameters, the 
equivalent forms, and the operator expressions as well as some reverses.

\section{Some Lemmas}

In the following lemmas, we assumed that $\alpha, \beta, \lambda>0, \alpha<$ $\lambda \beta, \alpha \leq \min \{1,2-\beta\}, \gamma_{1} \in(-\infty, \infty)$, and $0 \leq \gamma_{2} \leq 1 / 2$.

Lemma 1. Define the weight functions as follows:

$$
\begin{array}{r}
\omega_{\alpha}(n):=\left(n-\gamma_{2}\right)^{\alpha} \int_{\gamma_{1}}^{\infty} \frac{\left(x-\gamma_{1}\right)^{\alpha-1}}{\left[1+\left(x-\gamma_{1}\right)^{\beta}\left(n-\gamma_{2}\right)^{\beta}\right]^{\lambda}} d x \\
(n \in \mathbf{N}), \\
\omega_{\alpha}(x):=\left(x-\gamma_{1}\right)^{\alpha} \sum_{n=1}^{\infty} \frac{\left(n-\gamma_{2}\right)^{\alpha-1}}{\left[1+\left(x-\gamma_{1}\right)^{\beta}\left(n-\gamma_{2}\right)^{\beta}\right]^{\lambda}} \\
\left(x \in\left(\gamma_{1}, \infty\right)\right) .
\end{array}
$$

Setting $k:=k(\alpha, \beta, \lambda)=(1 / \beta) B(\alpha / \beta, \lambda-\alpha / \beta)$, one has the following inequalities:

$$
0<k(1-\theta(x))<\omega_{\alpha}(x)<\omega_{\alpha}(n)=k,
$$

where $\theta(x):=(1 / \beta k) \int_{0}^{\left(x-\gamma_{1}\right)^{\beta}\left(1-\gamma_{2}\right)^{\beta}}\left(u^{\alpha / \beta-1} /(1+u)^{\lambda}\right) d u \in$ $(0,1)$ and $\theta(x)=O\left(\left(x-\gamma_{1}\right)^{\alpha}\right)\left(x \in\left(\gamma_{1}, \infty\right)\right)$.

Proof. Putting $u=\left(x-\gamma_{1}\right)^{\beta}\left(n-\gamma_{2}\right)^{\beta}$ in (7), we have

$$
\omega_{\alpha}(n)=\frac{1}{\beta} \int_{0}^{\infty} \frac{u^{\alpha / \beta-1}}{(1+u)^{\lambda}} d u=\frac{1}{\beta} B\left(\frac{\alpha}{\beta}, \lambda-\frac{\alpha}{\beta}\right)=k .
$$

For fixed $x \in\left(\gamma_{1}, \infty\right)$, setting

$$
f(t):=\frac{\left(x-\gamma_{1}\right)^{\alpha}\left(t-\gamma_{2}\right)^{\alpha-1}}{\left[1+\left(x-\gamma_{1}\right)^{\beta}\left(t-\gamma_{2}\right)^{\beta}\right]^{\lambda}} \quad\left(t \in\left(\gamma_{2}, \infty\right)\right)
$$

in view of the conditions, we find that $f^{\prime}(t)=-(1-\alpha)(x-$ $\left.\gamma_{1}\right)^{\alpha}\left(t-\gamma_{2}\right)^{\alpha-2} /\left[1+\left(x-\gamma_{1}\right)^{\beta}\left(t-\gamma_{2}\right)^{\beta}\right]^{\lambda}-\lambda \beta\left(x-\gamma_{1}\right)^{\alpha+\beta}(t-$ $\left.\gamma_{2}\right)^{\alpha+\beta-2} /\left[1+\left(x-\gamma_{1}\right)^{\beta}\left(t-\gamma_{2}\right)^{\beta}\right]^{\lambda+1}<0$ and $f^{\prime \prime}(t)>0$. By the following Hadamard inequality (cf. [15]):

$$
f(n)<\int_{n-1 / 2}^{n+1 / 2} f(t) d t \quad(n \in \mathbf{N}),
$$

and letting $u=\left(x-\gamma_{1}\right)^{\beta}\left(t-\gamma_{2}\right)^{\beta}$, it follows that

$$
\begin{aligned}
\omega_{\alpha}(x) & =\sum_{n=1}^{\infty} f(n)<\sum_{n=1}^{\infty} \int_{n-1 / 2}^{n+1 / 2} f(t) d t=\int_{1 / 2}^{\infty} f(t) d t \\
& \leq \int_{\gamma_{2}}^{\infty} f(t) d t=\frac{1}{\beta} \int_{0}^{\infty} \frac{u^{\alpha / \beta-1}}{(1+u)^{\lambda}} d u \\
& =\frac{1}{\beta} B\left(\frac{\alpha}{\beta}, \lambda-\frac{\alpha}{\beta}\right)=k, \\
\omega_{\alpha}(x) & =\sum_{n=1}^{\infty} f(n)>\int_{1}^{\infty} f(t) d t \\
& =\int_{\gamma_{2}}^{\infty} f(t) d t-\int_{\gamma_{2}}^{1} f(t) d t \\
& =k-\frac{1}{\beta} \int_{0}^{\left(x-\gamma_{1}\right)^{\beta}\left(1-\gamma_{2}\right)^{\beta}} \frac{u^{\alpha / \beta-1}}{(1+u)^{\lambda}} d u \\
& =k(1-\theta(x))>0,
\end{aligned}
$$

where

$$
\begin{aligned}
0<\theta(x) & =\frac{1}{\beta k} \int_{0}^{\left(x-\gamma_{1}\right)^{\beta}\left(1-\gamma_{2}\right)^{\beta}} \frac{u^{\alpha / \beta-1}}{(1+u)^{\lambda}} d u \\
& <\frac{1}{\beta k} \int_{0}^{\left(x-\gamma_{1}\right)^{\beta}\left(1-\gamma_{2}\right)^{\beta}} u^{\alpha / \beta-1} d u=\frac{\left(1-\gamma_{2}\right)^{\alpha}\left(x-\gamma_{1}\right)^{\alpha}}{\alpha k} .
\end{aligned}
$$

Hence, we prove that (9) is valid.

Lemma 2. Suppose that $1 / p+1 / q=1(p \neq 0,1)$, and $a_{n} \geq 0$, and $f(x) \geq 0$ is a real measurable function in $\left(\gamma_{1}, \infty\right)$. Then the following are considered. (i) For $p>1$, one has the following:

$$
J:=\left\{\sum_{n=1}^{\infty}\left(n-\gamma_{2}\right)^{p \alpha-1}\right.
$$

$$
\left.\times\left[\int_{\gamma_{1}}^{\infty} \frac{f(x)}{\left[1+\left(x-\gamma_{1}\right)^{\beta}\left(n-\gamma_{2}\right)^{\beta}\right]^{\lambda}} d x\right]^{p}\right\}^{1 / p}
$$

$$
\leq k^{1 / q}\left\{\int_{\gamma_{1}}^{\infty} \omega_{\alpha}(x)\left(x-\gamma_{1}\right)^{p(1-\alpha)-1} f^{p}(x) d x\right\}^{1 / p},
$$




$$
\begin{aligned}
L_{1}:= & \left\{\int_{\gamma_{1}}^{\infty} \frac{\left(x-\gamma_{1}\right)^{q \alpha-1}}{\omega_{\alpha}^{q-1}(x)}\right. \\
& \left.\times\left[\sum_{n=1}^{\infty} \frac{a_{n}}{\left[1+\left(x-\gamma_{1}\right)^{\beta}\left(n-\gamma_{2}\right)^{\beta}\right]^{\lambda}}\right]^{q} d x\right\}^{1 / p} \\
\leq & \left\{k \sum_{n=1}^{\infty}\left(n-\gamma_{2}\right)^{q(1-\alpha)-1} a_{n}^{q}\right\}^{1 / q},
\end{aligned}
$$

where $\omega_{\alpha}(x)$ and $\omega_{\alpha}(n)$ are indicated by (7) and (8).

(ii) For $p<1(p \neq 0)$, one has the reverses of (15) and (16).

Proof. (i) By (7)-(9) and Hölder's inequality (cf. [15]), we find that

$$
\begin{aligned}
& {\left[\int_{\gamma_{1}}^{\infty} \frac{f(x)}{\left[1+\left(x-\gamma_{1}\right)^{\beta}\left(n-\gamma_{2}\right)^{\beta}\right]^{\lambda}} d x\right]^{p}} \\
& =\left\{\int_{\gamma_{1}}^{\infty} \frac{1}{\left[1+\left(x-\gamma_{1}\right)^{\beta}\left(n-\gamma_{2}\right)^{\beta}\right]^{\lambda}}\right. \\
& \times\left[\frac{\left(x-\gamma_{1}\right)^{(1-\alpha) / q}}{\left(n-\gamma_{2}\right)^{(1-\alpha) / p}} f(x)\right] \\
& \left.\times\left[\frac{\left(n-\gamma_{2}\right)^{(1-\alpha) / p}}{\left(x-\gamma_{1}\right)^{(1-\alpha) / q}}\right] d x\right\}^{p} \\
& \leq \int_{\gamma_{1}}^{\infty} \frac{1}{\left[1+\left(x-\gamma_{1}\right)^{\beta}\left(n-\gamma_{2}\right)^{\beta}\right]^{\lambda}} \\
& \times \frac{\left(x-\gamma_{1}\right)^{(1-\alpha)(p-1)}}{\left(n-\gamma_{2}\right)^{1-\alpha}} f^{p}(x) d x \\
& \times\left[\int_{\gamma_{1}}^{\infty} \frac{1}{\left[1+\left(x-\gamma_{1}\right)^{\beta}\left(n-\gamma_{2}\right)^{\beta}\right]^{\lambda}}\right. \\
& \left.\times \frac{\left(n-\gamma_{2}\right)^{(1-\alpha)(q-1)}}{\left(x-\gamma_{1}\right)^{1-\alpha}} d x\right]^{p-1}
\end{aligned}
$$

$$
\begin{gathered}
J^{p} \leq k^{p^{-1}} \sum_{n=1}^{\infty} \int_{\gamma_{1}}^{\infty} \frac{f^{p}(x)}{\left[1+\left(x-\gamma_{1}\right)^{\beta}\left(n-\gamma_{2}\right)^{\beta}\right]^{\lambda}} \\
\quad \times \frac{\left(x-\gamma_{1}\right)^{(1-\alpha)(p-1)}}{\left(n-\gamma_{2}\right)^{1-\alpha}} d x \\
=k^{p-1} \int_{\gamma_{1}}^{\infty} \sum_{n=1}^{\infty} \frac{\left(n-\gamma_{2}\right)^{\alpha-1}}{\left[1+\left(x-\gamma_{1}\right)^{\beta}\left(n-\gamma_{2}\right)^{\beta}\right]^{\lambda}} \\
=k^{p-1} \int_{\gamma_{1}}^{\infty} \varrho_{\alpha}(x)\left(x-\gamma_{1}\right)^{p(1-\alpha)-1} f^{p}(x) d x .
\end{gathered}
$$

Hence, (15) is valid. Using Hölder's inequality again, we have

$$
\begin{aligned}
{\left[\sum_{n=1}^{\infty}\right.} & \left.\frac{a_{n}}{\left[1+\left(x-\gamma_{1}\right)^{\beta}\left(n-\gamma_{2}\right)^{\beta}\right]^{\lambda}}\right]^{q} \\
= & \left\{\sum_{n=1}^{\infty} \frac{1}{\left[1+\left(x-\gamma_{1}\right)^{\beta}\left(n-\gamma_{2}\right)^{\beta}\right]^{\lambda}}\right. \\
& \times\left[\frac{\left(x-\gamma_{1}\right)^{(1-\alpha) / q}}{\left.\left(n-\gamma_{2}\right)^{(1-\alpha) / p}\right]\left[\frac{\left(n-\gamma_{2}\right)^{(1-\alpha) / p}}{\left(x-\gamma_{1}\right)^{(1-\alpha) / q}} a_{n}\right]}\right]^{q} \\
\leq & {\left[\Phi_{\alpha}(x)\left(x-\gamma_{1}\right)^{p(1-\alpha)-1}\right]^{q-1} } \\
& \times \sum_{n=1}^{\infty} \frac{a_{n}^{q}}{\left[1+\left(x-\gamma_{1}\right)^{\beta}\left(n-\gamma_{2}\right)^{\beta}\right]^{\lambda}} \frac{\left(n-\gamma_{2}\right)^{(1-\alpha) q / p}}{\left(x-\gamma_{1}\right)^{1-\alpha}} \\
= & \omega_{\alpha}^{q-1}(x)\left(x-\gamma_{1}\right)^{1-q \alpha} \\
& \times \sum_{n=1}^{\infty} \frac{\left(n-\gamma_{2}\right)^{(1-\alpha)(q-1)}}{\left[1+\left(x-\gamma_{1}\right)^{\beta}\left(n-\gamma_{2}\right)^{\beta}\right]^{\lambda}} \frac{1}{\left(x-\gamma_{1}\right)^{1-\alpha}} a_{n}^{q},
\end{aligned}
$$




$$
\begin{aligned}
L_{1}^{q} \leq \int_{\gamma_{1}}^{\infty} \sum_{n=1}^{\infty} \frac{1}{\left[1+\left(x-\gamma_{1}\right)^{\beta}\left(n-\gamma_{2}\right)^{\beta}\right]^{\lambda}} \\
\quad \times \frac{\left(n-\gamma_{2}\right)^{(1-\alpha)(q-1)}}{\left(x-\gamma_{1}\right)^{1-\alpha}} a_{n}^{q} d x \\
=\sum_{n=1}^{\infty}\left[\left(n-\gamma_{2}\right)^{\alpha} \int_{\gamma_{1}}^{\infty} \frac{\left(x-\gamma_{1}\right)^{\alpha-1}}{\left[1+\left(x-\gamma_{1}\right)^{\beta}\left(n-\gamma_{2}\right)^{\beta}\right]^{\lambda}} d x\right] \\
=\sum_{n=1}^{\infty} \omega_{\alpha}(n)\left(n-\gamma_{2}\right)^{q(1-\alpha)-1} a_{n}^{q} \\
=k \sum_{n=1}^{\infty}\left(n-\gamma_{2}\right)^{q(1-\alpha)-1} a_{n}^{q} .
\end{aligned}
$$

Hence, (16) is valid.

(ii) For $0<p<1(q<0)$ or $p<0(0<q<1)$, using the reverse Hölder inequality and in the same way, we have the reverses of (15) and (16).

\section{Main Results}

Some functions and spaces are introduced as follow:

$$
\begin{aligned}
& \phi(x):=\left(x-\gamma_{1}\right)^{p(1-\alpha)-1} \\
& \widetilde{\phi}(x):=(1-\theta(x)) \phi(x) \\
& \psi(n):=\left(n-\gamma_{2}\right)^{q(1-\alpha)-1}
\end{aligned}
$$

$\left(x \in\left(\gamma_{1}, \infty\right), n \in \mathbf{N}, \theta(x)\right.$ is indicated as Lemma 1$)$,

$$
\begin{gathered}
L_{p, \phi}\left(\gamma_{1}, \infty\right):=\left\{f ;\|f\|_{p, \phi}=\left\{\int_{\gamma_{1}}^{\infty} \phi(x)|f(x)|^{p} d x\right\}^{1 / p}<\infty\right\}, \\
l_{q, \psi}:=\left\{a=\left\{a_{n}\right\}_{n=1}^{\infty} ;\|a\|_{q, \psi}=\left\{\sum_{n=1}^{\infty} \psi(n)\left|a_{n}\right|^{q}\right\}^{1 / q}<\infty\right\} .
\end{gathered}
$$

Note. If $p>1$, then $L_{p, \phi}\left(\gamma_{1}, \infty\right)$ and $l_{q, \psi}$ are normal spaces; if $0<p<1$ or $p<0$, then both $L_{p, \phi}\left(\gamma_{1}, \infty\right)$ and $l_{q, \psi}$ are not normal spaces, but we still use the formal symbols in the following.

Theorem 3. Suppose that $p>1,1 / p+1 / q=1, \alpha, \beta, \lambda>$ $0, \alpha<\lambda \beta, \alpha \leq \min \{1,2-\beta\}, \gamma_{1} \in(-\infty, \infty), 0 \leq$ $\gamma_{2} \leq 1 / 2$, and $f(x), a_{n} \geq 0$, such that $f \in L_{p, \phi}\left(\gamma_{1}, \infty\right)$, $a=\left\{a_{n}\right\}_{n=1}^{\infty} \in l_{q, \psi},\|f\|_{p, \phi}>0$, and $\|a\|_{q, \psi}>0$, then we have the following equivalent inequalities:

$$
\begin{aligned}
I & :=\sum_{n=1}^{\infty} a_{n} \int_{\gamma_{1}}^{\infty} \frac{f(x)}{\left[1+\left(x-\gamma_{1}\right)^{\beta}\left(n-\gamma_{2}\right)^{\beta}\right]^{\lambda}} d x \\
& =\int_{\gamma_{1}}^{\infty} f(x) \sum_{n=1}^{\infty} \frac{a_{n} d x}{\left[1+\left(x-\gamma_{1}\right)^{\beta}\left(n-\gamma_{2}\right)^{\beta}\right]^{\lambda}} \\
& <k\|f\|_{p, \phi}\|a\|_{q, \psi}, \\
J & =\left\{\sum_{n=1}^{\infty}\left(n-\gamma_{2}\right)^{p \alpha-1}\left[\int_{\gamma_{1}}^{\infty} \frac{f(x) d x}{\left[1+\left(x-\gamma_{1}\right)^{\beta}\left(n-\gamma_{2}\right)^{\beta}\right]^{\lambda}}\right]^{p}\right\}^{1 / p} \\
& <k\|f\|_{p, \phi},
\end{aligned}
$$

$$
\begin{aligned}
L & =\left\{\int_{\gamma_{1}}^{\infty}\left(x-\gamma_{1}\right)^{q \alpha-1}\left[\sum_{n=1}^{\infty} \frac{a_{n}}{\left[1+\left(x-\gamma_{1}\right)^{\beta}\left(n-\gamma_{2}\right)^{\beta}\right]^{\lambda}}\right]^{q} d x\right\}^{1 / q} \\
& <k\|a\|_{q, \psi},
\end{aligned}
$$

where the constant factor $k=(1 / \beta) B(\alpha / \beta, \lambda-\alpha / \beta)$ is the best possible.

Proof. By the Lebesgue term-by-term integration theorem, we find that there are two expressions of $I$ in (21). In view of (15), $\omega_{\alpha}(x)<k$, and $0<\|f\|_{p, \phi}<\infty$, we have (22). By Hölder's inequality, we find that

$$
\begin{aligned}
I=\sum_{n=1}^{\infty} & {\left[\left(n-\gamma_{2}\right)^{\alpha-1 / p} \int_{\gamma_{1}}^{\infty} \frac{f(x) d x}{\left[1+\left(x-\gamma_{1}\right)^{\beta}\left(n-\gamma_{2}\right)^{\beta}\right]^{\lambda}}\right] } \\
& \times\left[\left(n-\gamma_{2}\right)^{1 / p-\alpha} a_{n}\right] \\
\leq J & \left\{\sum_{n=1}^{\infty}\left(n-\gamma_{2}\right)^{q(1-\alpha)-1} a_{n}^{q}\right\}^{1 / q}=J\|a\|_{q, \psi} .
\end{aligned}
$$

Hence, (21) is valid by (22). On the other hand, assuming that (21) is valid and setting

$$
\begin{array}{r}
a_{n}:=\left(n-\gamma_{2}\right)^{p \alpha-1}\left[\int_{\gamma_{1}}^{\infty} \frac{f(x)}{\left[1+\left(x-\gamma_{1}\right)^{\beta}\left(n-\gamma_{2}\right)^{\beta}\right]^{\lambda}} d x\right]_{(n \in \mathbf{N}),}^{p-1} \\
\end{array}
$$

then we have

$$
\|a\|_{q, \psi}^{q}=\sum_{n=1}^{\infty}\left(n-\gamma_{2}\right)^{q(1-\alpha)-1} a_{n}^{q}=J^{p}=I .
$$


By (15), it follows that $J<\infty$. If $J=0$, then (22) is trivially valid. If $J>0$, then $0<\|a\|_{q, \psi}=J^{p-1}<\infty$. By (21), we have

$$
\begin{aligned}
& \|a\|_{q, \psi}^{q}=J^{p}=I<k\|f\|_{p, \phi}\|a\|_{q, \psi}, \\
& \text { that is, } \quad J=\|a\|_{q, \psi}^{q-1}<k\|f\|_{p, \phi} .
\end{aligned}
$$

Hence, (22) is valid, which is equivalent to (21).

In view of (16) and $\omega_{\alpha}(x)<k$, we obtain (23). By Hölder's inequality again, we have

$$
\begin{aligned}
I= & \int_{\gamma_{1}}^{\infty}\left[\left(x-\gamma_{1}\right)^{\alpha-1 / q} \sum_{n=1}^{\infty} \frac{a_{n}}{\left[1+\left(x-\gamma_{1}\right)^{\beta}\left(n-\gamma_{2}\right)^{\beta}\right]^{\lambda}}\right] \\
& \times\left[\left(x-\gamma_{1}\right)^{1 / q-\alpha} f(x)\right] d x \\
\leq & L\left\{\int_{\gamma_{1}}^{\infty}\left(x-\gamma_{1}\right)^{p(1-\alpha)-1} f^{p}(x) d x\right\}^{1 / p}=L\|f\|_{p, \phi} .
\end{aligned}
$$

Then (21) is valid by using (23). Supposing that (21) is valid and setting

$$
\begin{array}{r}
f(x):=\left(x-\gamma_{1}\right)^{q \alpha-1}\left[\sum_{n=1}^{\infty} \frac{a_{n}}{\left[1+\left(x-\gamma_{1}\right)^{\beta}\left(n-\gamma_{2}\right)^{\beta}\right]^{\lambda}}\right]^{q-1} \\
\left(x \in\left(\gamma_{1}, \infty\right)\right),
\end{array}
$$

then it follows that

$$
\|f\|_{p, \phi}^{p}=\int_{\gamma_{1}}^{\infty}\left(x-\gamma_{1}\right)^{p(1-\alpha)-1} f^{p}(x) d x=L^{q}=I .
$$

In view of (16) and (9), we find $L<\infty$. If $L=0$, then (23) is trivially valid; if $L>0$, that is, $0<\|f\|_{p, \phi}<\infty$, then, by (21), we have

$$
\begin{aligned}
& \|f\|_{p, \phi}^{p}=L^{q}=I<k\|f\|_{p, \phi}\|a\|_{q, \psi}, \\
& \text { that is, } \quad L=\|f\|_{p, \phi}^{p-1}<k\|a\|_{q, \psi} .
\end{aligned}
$$

Hence (23) is valid, which is equivalent to (21). Then (21), (22), and (23) are equivalent. We prove that the constant factor in (21) is the best possible. For $0<\varepsilon<q \alpha$, setting $\widetilde{a}=\left\{\widetilde{a}_{n}\right\}_{n=1}^{\infty}$ and $\widetilde{f}(x)$ as follows $(p, q>1)$ :

$$
\begin{gathered}
\tilde{a}_{n}=\left(n-\gamma_{2}\right)^{\alpha-\varepsilon / q-1}, \\
\tilde{f}(x)= \begin{cases}\left(x-\gamma_{1}\right)^{\alpha+\varepsilon / p-1}, & x \in\left(\gamma_{1}, 1+\gamma_{1}\right), \\
0, & x \in\left[1+\gamma_{1}, \infty\right),\end{cases}
\end{gathered}
$$

if there exists a positive number $k^{\prime} \leq k$, such that (21) is still valid as we replace $k$ by $k^{\prime}$, then, substitution of $\tilde{a}$ and $\tilde{f}(x)$, we have

$$
\begin{aligned}
\widetilde{I} & :=\sum_{n=1}^{\infty} \widetilde{a}_{n} \int_{\gamma_{1}}^{\infty} \frac{\tilde{f}(x)}{\left[1+\left(x-\gamma_{1}\right)^{\beta}\left(n-\gamma_{2}\right)^{\beta}\right]^{\lambda}} d x \\
& <k^{\prime}\|\tilde{f}\|_{p, \phi}\|\tilde{a}\|_{q, \psi} .
\end{aligned}
$$

For $p, q>1$, letting $\widetilde{\alpha}=\alpha-\varepsilon / q$, we find by Lemma 1 that

$$
\begin{aligned}
\widetilde{I}= & \int_{\gamma_{1}}^{\gamma_{1}+1}\left(x-\gamma_{1}\right)^{\varepsilon-1}\left(x-\gamma_{1}\right)^{\alpha-\varepsilon / q} \\
& \times \sum_{n=1}^{\infty} \frac{\left(n-\gamma_{2}\right)^{\alpha-\varepsilon / q-1} d x}{\left[1+\left(x-\gamma_{1}\right)^{\beta}\left(n-\gamma_{2}\right)^{\beta}\right]^{\lambda}} \\
= & \int_{\gamma_{1}}^{\gamma_{1}+1}\left(x-\gamma_{1}\right)^{\varepsilon-1} \omega_{\widetilde{\alpha}}(x) d x \\
> & k(\widetilde{\alpha}, \beta, \lambda) \int_{\gamma_{1}}^{\gamma_{1}+1}\left(x-\gamma_{1}\right)^{\varepsilon-1}\left[1-O\left(\left(x-\gamma_{1}\right)^{\widetilde{\alpha}}\right)\right] d x \\
= & k\left(\alpha-\frac{\varepsilon}{q}, \beta, \lambda\right)\left(\frac{1}{\varepsilon}-O(1)\right) .
\end{aligned}
$$

By simple calculation, we have

$$
\begin{aligned}
\|\widetilde{a}\|_{q, \psi}^{q} & =\sum_{n=1}^{\infty}\left(n-\gamma_{2}\right)^{q(1-\alpha)-1} \widetilde{a}_{n}^{q}=\sum_{n=1}^{\infty}\left(n-\gamma_{2}\right)^{-1-\varepsilon} \\
& <\left(1-\gamma_{2}\right)^{-1-\varepsilon}+\int_{1}^{\infty}\left(y-\gamma_{2}\right)^{-1-\varepsilon} d y=\frac{\varepsilon+1-\gamma_{2}}{\varepsilon\left(1-\gamma_{2}\right)^{\varepsilon+1}},
\end{aligned}
$$

$$
\|\widetilde{f}\|_{p, \phi}=\left\{\int_{\gamma_{1}}^{\gamma_{1}+1}\left(x-\gamma_{1}\right)^{\varepsilon-1} d x\right\}^{1 / p}=\left(\frac{1}{\varepsilon}\right)^{1 / p} .
$$

In view of (33), (34), and $q>1$, it follows that

$$
\begin{aligned}
k(\alpha & \left.-\frac{\varepsilon}{q}, \beta, \lambda\right)[1-\varepsilon O(1)] \\
& <\varepsilon \widetilde{I}<k^{\prime}\|\widetilde{f}\|_{p, \phi}\|\widetilde{a}\|_{q, \psi}<k^{\prime}\left[\frac{\varepsilon+1-\gamma_{2}}{\left(1-\gamma_{2}\right)^{\varepsilon+1}}\right]^{1 / q} .
\end{aligned}
$$

Then we have $k(\alpha, \beta, \lambda)=k \leq k^{\prime}$ for $\varepsilon \rightarrow 0^{+}$. Hence, $k^{\prime}=k$ is the best value of (21). By the equivalency, the constant factor $k$ in $(22)((23))$ is the best possible. Otherwise, we would reach a contradiction by $(24)((28))$ that the constant factor in (21) is not the best possible.

Remark 4. (i) Define a half-discrete Hilbert operator $T$ : $L_{p, \phi}\left(\gamma_{1}, \infty\right) \rightarrow l_{p, \psi^{1-p}}$ as follows. For $f \in L_{p, \phi}\left(\gamma_{1}, \infty\right)$, we define $T f \in l_{p, \psi^{1-p}}$, satisfying

$$
T f(n)=\int_{\gamma_{1}}^{\infty} \frac{f(x)}{\left[1+\left(x-\gamma_{1}\right)^{\beta}\left(n-\gamma_{2}\right)^{\beta}\right]^{\lambda}} d x \quad(n \in \mathbf{N})
$$


Then by (22), it follows that $\|T f\|_{p, \psi^{1-p}} \leq k\|f\|_{p, \phi}$; that is, $T$ is the bounded operator with $\|T\| \leq k$. Since the constant factor $k$ in (22) is the best possible, then we have $\|T\|=k=$ $(1 / \beta) B(\alpha / \beta, \lambda-\alpha / \beta)$.

(ii) Define a half-discrete Hilbert operator $\widetilde{T}: l_{q, \psi} \rightarrow$ $L_{q, \phi^{1-q}}\left(\gamma_{1}, \infty\right)$ in the following way. For $a \in l_{q, \psi}$, we define $\widetilde{T} a \in L_{q, \phi^{1-q}}$, satisfying

$$
\widetilde{T} a(x)=\sum_{n=1}^{\infty} \frac{a_{n}}{\left[1+\left(x-\gamma_{1}\right)^{\beta}\left(n-\gamma_{2}\right)^{\beta}\right]^{\lambda}} \quad\left(x \in\left(\gamma_{1}, \infty\right)\right) .
$$

Then by (23), it follows that $\|\widetilde{T} a\|_{q, \phi^{1-q}} \leq k\|a\|_{q, \psi}$; that is, $\widetilde{T}$ is the bounded operator with $\|\widetilde{T}\| \leq k$. Since the constant factor $k$ in (23) is the best possible, then we have $\|\widetilde{T}\|=k=$ $(1 / \beta) B(\alpha / \beta, \lambda-\alpha / \beta)$.

Theorem 5. Suppose that $0<p<1,1 / p+1 / q=1, \alpha, \beta, \lambda>$ $0, \alpha<\lambda \beta, \alpha \leq \min \{1,2-\beta\}, \gamma_{1} \in(-\infty, \infty), 0 \leq \gamma_{2} \leq 1 / 2$, and $f(x), a_{n} \geq 0$, such that $f \in L_{p, \tilde{\phi}}\left(\gamma_{1}, \infty\right), a=\left\{a_{n}\right\}_{n=1}^{\infty} \in$ $l_{q, \psi},\|f\|_{p, \tilde{\phi}}>0$, and $\|a\|_{q, \psi}>0$. Then one has the following equivalent inequalities:

$$
\begin{aligned}
I & =\sum_{n=1}^{\infty} a_{n} \int_{\gamma_{1}}^{\infty} \frac{f(x)}{\left[1+\left(x-\gamma_{1}\right)^{\beta}\left(n-\gamma_{2}\right)^{\beta}\right]^{\lambda}} d x \\
& =\int_{\gamma_{1}}^{\infty} f(x) \sum_{n=1}^{\infty} \frac{a_{n} d x}{\left[1+\left(x-\gamma_{1}\right)^{\beta}\left(n-\gamma_{2}\right)^{\beta}\right]^{\lambda}} \\
& >k\|f\|_{p, \tilde{\phi}}\|a\|_{q, \psi}, \\
J & =\left\{\sum_{n=1}^{\infty}\left(n-\gamma_{2}\right)^{p \alpha-1}\left[\int_{\gamma_{1}}^{\infty} \frac{f\left(1+\left(x-\gamma_{1}\right)^{\beta}\left(n-\gamma_{2}\right)^{\beta}\right]^{\lambda}}{[1 x}\right]^{p}\right\}^{1 / p} \\
& >k\|f\|_{p, \tilde{\phi},} \\
\widetilde{L} & :=\left\{\int_{\gamma_{1}}^{\infty} \frac{\left(x-\gamma_{1}\right)^{q \alpha-1}}{[1-\theta(x)]^{q-1}}\left[\sum_{n=1}^{\infty} \frac{a_{n}\left[1+\left(x-\gamma_{1}\right)^{\beta}\left(n-\gamma_{2}\right)^{\beta}\right]^{\lambda}}{[1-} d x\right\}^{1 / q}\right. \\
& >k\|a\|_{q, \psi},
\end{aligned}
$$

where the constant factor $k=(1 / \beta) B(\alpha / \beta, \lambda-\alpha / \beta)$ is the best possible.

Proof. In view of (9), the reverse of (15), and $0<\|f\|_{p, \tilde{\phi}}<\infty$, we have (41). Using the reverse Hölder inequality, we obtain the reverse form of (24) as follows:

$$
I \geq J\|a\|_{q, \psi} .
$$

Then by (41), (40) is valid.
On the other hand, if (40) is valid, setting $a_{n}$ as in (25), then (26) still holds with $0<p<1$. By (40), it follows that $J>0$. If $J=\infty$, then (41) is trivially valid; if $J<\infty$, then $0<\|a\|_{q, \psi}=J^{p-1}<\infty$, and we have

$$
\begin{aligned}
& \|a\|_{q, \psi}^{q}=J^{p}=I>k\|f\|_{p, \tilde{\phi}}\|a\|_{q, \psi}, \\
& \text { that is, } \quad J=\|a\|_{q, \psi}^{q-1}>k\|f\|_{p, \tilde{\phi}} .
\end{aligned}
$$

Then (41) is valid, which is equivalent to (40).

By the reverse of $(16)$ and $\omega(x)>k(1-\theta(x))$, and by $q<0$, we have

$$
\begin{aligned}
\tilde{L}>k^{(q-1) / q} L_{1} & \geq k^{(q-1) / q}\left\{k \sum_{n=1}^{\infty}\left(n-\gamma_{2}\right)^{q(1-\alpha)-1} a_{n}^{q}\right\}^{1 / q} \\
& =k\|a\|_{q, \psi} .
\end{aligned}
$$

Hence, (42) is valid. By the reverse Hölder inequality again, we obtain

$$
\begin{aligned}
I= & \int_{\gamma_{1}}^{\infty}\left[\frac{\left(x-\gamma_{1}\right)^{\alpha-1 / q}}{(1-\theta(x))^{1 / p}} \sum_{n=1}^{\infty} \frac{a_{n}}{\left[1+\left(x-\gamma_{1}\right)^{\beta}\left(n-\gamma_{2}\right)^{\beta}\right]^{\lambda}}\right] \\
& \times\left[(1-\theta(x))^{1 / p}\left(x-\gamma_{1}\right)^{1 / q-\alpha} f(x)\right] d x \\
\geq & \widetilde{L}\|f\|_{p, \tilde{\phi}} .
\end{aligned}
$$

Then (40) is valid by (42). On the other hand, if (40) is valid, setting

$$
\begin{array}{r}
f(x)=\frac{\left(x-\gamma_{1}\right)^{q \alpha-1}}{[1-\theta(x)]^{q-1}}\left[\sum_{n=1}^{\infty} \frac{a_{n}}{\left[1+\left(x-\gamma_{1}\right)^{\beta}\left(n-\gamma_{2}\right)^{\beta}\right]^{\lambda}}\right]^{q-1} \\
\left(x \in\left(\gamma_{1}, \infty\right)\right),
\end{array}
$$

then $\|f\|_{p, \tilde{\phi}}^{p}=\int_{\gamma_{1}}^{\infty}[1-\theta(x)]\left(x-\gamma_{1}\right)^{p(1-\alpha)-1} f^{p}(x) d x=\widetilde{L}^{q}=I$. By the reverse of (16), we have $\widetilde{L}>0$. If $\widetilde{L}=\infty$, then (42) is trivially valid; if $0<\widetilde{L}<\infty$, then, by (40), we obtain

$$
\begin{gathered}
\|f\|_{p, \tilde{\phi}}^{p}=\widetilde{L}^{q}=I>k\|f\|_{p, \tilde{\phi}}\|a\|_{q, \psi}, \\
\text { that is, } \widetilde{L}=\|f\|_{p, \widetilde{\phi}}^{p-1}>k\|a\|_{q, \psi} .
\end{gathered}
$$

Hence, (42) is valid, which is equivalent to (40). It follows that (40), (41), and (42) are equivalent. 
If there exists a positive number $k^{\prime} \geq k$, such that (40) is still valid as we replace $k$ by $k^{\prime}$, then, in particular, we have

$$
\begin{aligned}
\widetilde{I} & =\sum_{n=1}^{\infty} \tilde{a}_{n} \int_{\gamma_{1}}^{\infty} \frac{\tilde{f}(x)}{\left[1+\left(x-\gamma_{1}\right)^{\beta}\left(n-\gamma_{2}\right)^{\beta}\right]^{\lambda}} d x \\
& >k^{\prime}\|\tilde{f}\|_{p, \tilde{\phi}}\|\tilde{a}\|_{q, \psi},
\end{aligned}
$$

where $\tilde{a}=\left\{\tilde{a}_{n}\right\}_{n=1}^{\infty}$ and $\tilde{f}$ are taken as in (32) $(0<\varepsilon<p(\beta \lambda-$ $\alpha)$ ). Since, by (35), we find in

$$
\begin{aligned}
\|\tilde{f}\|_{p, \tilde{\phi}} & =\left\{\int_{\gamma_{1}}^{1+\gamma_{1}}\left[1-O\left(\left(x-\gamma_{1}\right)^{\alpha}\right)\right]\left(x-\gamma_{1}\right)^{\varepsilon-1} d x\right\}^{1 / p} \\
& =\left(\frac{1}{\varepsilon}-O(1)\right)^{1 / p}, \\
\widetilde{I} & \leq \sum_{n=1}^{\infty}\left(n-\gamma_{2}\right)^{\alpha-\varepsilon / q-1} \int_{\gamma_{1}}^{\infty} \frac{\left(x-\gamma_{1}\right)^{\alpha+\varepsilon / p-1}}{\left[1+\left(x-\gamma_{1}\right)^{\beta}\left(n-\gamma_{2}\right)^{\beta}\right]^{\lambda}} d x \\
& =\frac{1}{\beta} \sum_{n=1}^{\infty}\left(n-\gamma_{2}\right)^{-\varepsilon-1} \int_{0}^{\infty} \frac{u^{\alpha / \beta+\varepsilon / \beta p-1}}{(1+u)^{\lambda}} d u \\
& \leq \frac{1}{\beta}\left[\frac{\varepsilon+1-\gamma_{2}}{\varepsilon\left(1-\gamma_{2}\right)^{\varepsilon+1}}\right] B\left(\frac{\alpha}{\beta}+\frac{\varepsilon}{\beta p}, \lambda-\frac{\alpha}{\beta}-\frac{\varepsilon}{\beta p}\right),
\end{aligned}
$$

then, by (35), (49), and the above results, we obtain (notice that $q<0$ )

$$
\begin{gathered}
\frac{1}{\beta}\left[\frac{\varepsilon+1-\gamma_{2}}{\left(1-\gamma_{2}\right)^{\varepsilon+1}}\right] B\left(\frac{\alpha}{\beta}+\frac{\varepsilon}{\beta p}, \lambda-\frac{\alpha}{\beta}-\frac{\varepsilon}{\beta p}\right) \\
\geq \varepsilon \tilde{I}>\varepsilon k^{\prime}\left(\frac{1}{\varepsilon}-O(1)\right)^{1 / p}\left(\frac{\varepsilon+1-\gamma_{2}}{\varepsilon\left(1-\gamma_{2}\right)^{\varepsilon+1}}\right)^{1 / q} \\
=k^{\prime}(1-\varepsilon O(1))^{1 / p}\left(\frac{\varepsilon+1-\gamma_{2}}{\left(1-\gamma_{2}\right)^{\varepsilon+1}}\right)^{1 / q} .
\end{gathered}
$$

For $\varepsilon \rightarrow 0^{+}$, we have $k=(1 / \beta) B(\alpha / \beta, \lambda-\alpha / \beta) \geq k^{\prime}$. Hence, $k=k^{\prime}$ is the best value of (40). By the equivalency, the constant factor $k$ in $(41)((42))$ is the best possible. Otherwise, we would reach a contradiction by $(43)((46))$ that the constant factor in (40) is not the best possible. result.

In the same way, for $p<0$, we also have the following

Theorem 6. If the assumption of $p>1$ in Theorem 3 is replaced by $p<0$, then the reverses of (21), (22), and (23) are valid and equivalent. Moreover, the same constant factor is the best possible.
Remark 7. (i) If $\gamma_{1}=\gamma_{2}=0, \lambda=\beta=1$, and $\alpha=1 / p$, then $k=B(1 / p, 1 / q)$ and (23) reduces to (3). In particular, for $p=q=2$, (21) reduces to (4).

(ii) For $\gamma_{1}=0, \gamma_{2}=1 / 2$, and $\alpha=1 / p$ in (21) and (23), it follows

$$
\begin{gathered}
\sum_{n=1}^{\infty} a_{n} \int_{0}^{\infty} \frac{f(x)}{\left(1+(x n-x / 2)^{\beta}\right)^{\lambda}} d x \\
<k\left\{\int_{0}^{\infty} x^{p-2} f^{p}(x) d x\right\}^{1 / p}\left\{\sum_{n=1}^{\infty} a_{n}^{q}\right\}^{1 / q}, \\
\int_{0}^{\infty} x^{q-2}\left(\sum_{n=1}^{\infty} \frac{a_{n}}{\left(1+(x n-x / 2)^{\beta}\right)^{\lambda}}\right)^{q} d x<k^{q} \sum_{n=1}^{\infty} a_{n}^{q},
\end{gathered}
$$

where the constant factors both $k=(1 / \beta) B(1 / p \beta, \lambda-1 / p \beta)$ and $k^{q}$ are the best possibles. In particular, for $\lambda=\beta=1$, (53) comes to (5); for $\lambda=\beta=1$ and $p=q=2$, in (52), we obtain (6). Hence, inequality (21) is the best extension of (4) and (6), and inequality (23) is the best extension of (3) and (5) with the parameters.

\section{Acknowledgment}

This paper is supported by the 2012 Knowledge Construction Special Foundation Item of Guangdong Institution of Higher Learning College and University (no. 2012KJCX0079).

\section{References}

[1] H. Weyl, Singulare integral gleichungen mit besonderer berucksichtigung des fourierschen integral theorems [InaugeralDissertation], Gottingen, 1908.

[2] I. Schur, "Bernerkungen sur Theorie der beschrankten Bilinearformen mit unendlich vielen veranderlichen," Journal für die Reine und Angewandte Mathematik, vol. 140, pp. 1-28, 1911.

[3] D. S. Mitrinović, J. E. Pečarić, and A. M. Fink, Inequalities Involving Functions and Their Integrals and Derivatives, Kluwer Acaremic, Boston, Mass, USA, 1991.

[4] Y. Bicheng, “On Hilbert's Integral Inequality," Journal of Mathematical Analysis and Applications, vol. 220, no. 2, pp. 778-785, 1998.

[5] B. Yang and L. Debnath, "On the extended Hardy-Hilbert's inequality," Journal of Mathematical Analysis and Applications, vol. 272, no. 1, pp. 187-199, 2002.

[6] J. Jin and L. Debnath, "On a Hilbert-type linear series operator and its applications," Journal of Mathematical Analysis and Applications, vol. 371, no. 2, pp. 691-704, 2010.

[7] B. Yang and T. M. Rassias, "On a new extension of Hilbert's inequality," Mathematical Inequalities and Applications, vol. 8, no. 4, pp. 575-582, 2005.

[8] M. Krnić and J. Pečarić, "Hilbert's inequalities and their reverses," Publicationes Mathematicae Debrecen, vol. 67, no. 34, pp. 315-331, 2005.

[9] L. E. Azar, "On some extensions of hardy-hilbert's inequality and applications," Journal of Inequalities and Applications, vol. 2008, Article ID 546829, 2008. 
[10] Q. Huang and B. Yang, "On a multiple hilbert-type integral operator and applications," Journal of Inequalities and Applications, vol. 2009, Article ID 192197, 2009.

[11] Q. Huang, "On a Multiple hilbert's inequality with parameters," Journal of Inequalities and Applications, vol. 2010, Article ID 309319, 2010.

[12] B. Yang, The Norm of Operator and Hilbert-Type Inequalities, Science Press, Beijing, China, 2009 (Chinese).

[13] B. Yang, Hilbert-Type Integral Inequalities, Bentham Science, Dubai, UAE, 2009.

[14] B. Yang, Discrete Hilbert-Type Inequalities, Bentham Science, Dubai, UAE, 2011.

[15] J. Kuang, Applied Inequalities, Shangdong Science Technic Press, Jinan, China, 2010 (Chinese).

[16] G. H. Hardy, J. E. Littlewood, and G. Pólya, Inequalities, Cambridge University Press, Cambridge, UK, 1934.

[17] B. Yang, "A mixed Hilbert-type inequality with a best constant factor," International Journal of Pure and Applied Mathematics, vol. 20, no. 3, pp. 319-328, 2005.

[18] B. Yang, "On a half-discrete reverse hilbert-yype inequality with a non-homogeneous kernel," Journal of Inner Mongolia Normal University (Natural Science Edition), vol. 40, no. 5, pp. 433-436, 2011 (Chinese). 


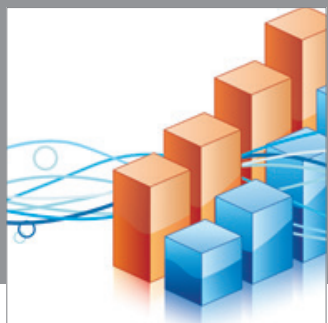

Advances in

Operations Research

mansans

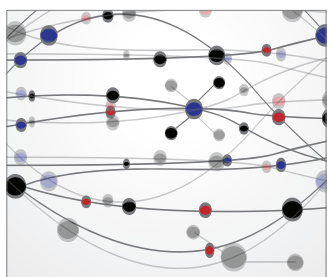

The Scientific World Journal
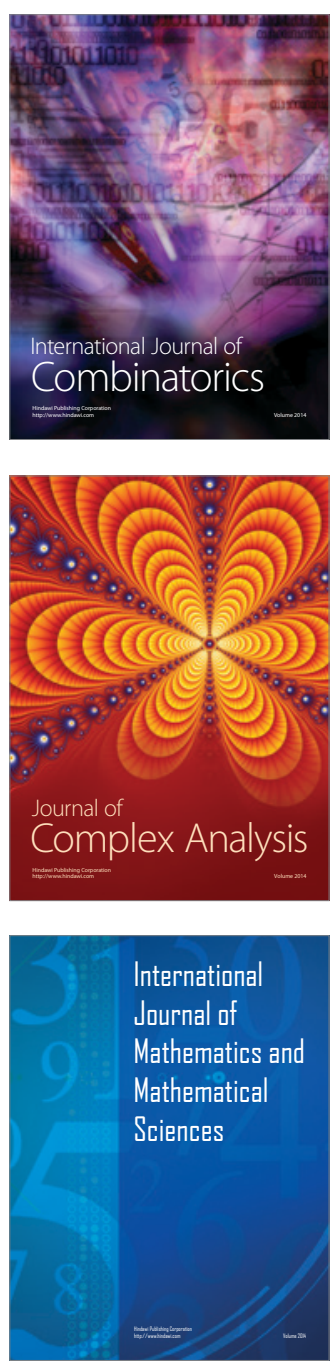
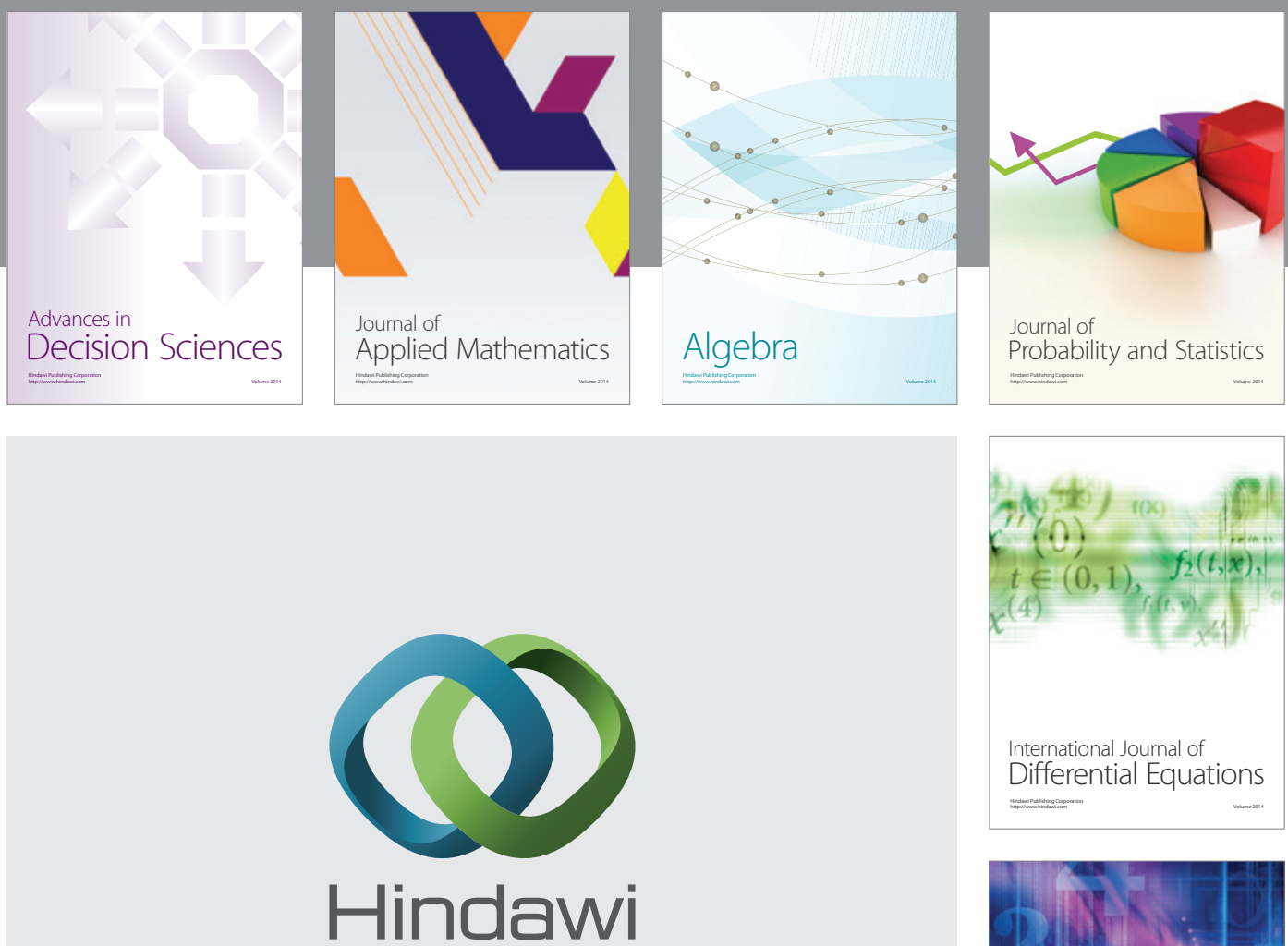

Submit your manuscripts at http://www.hindawi.com
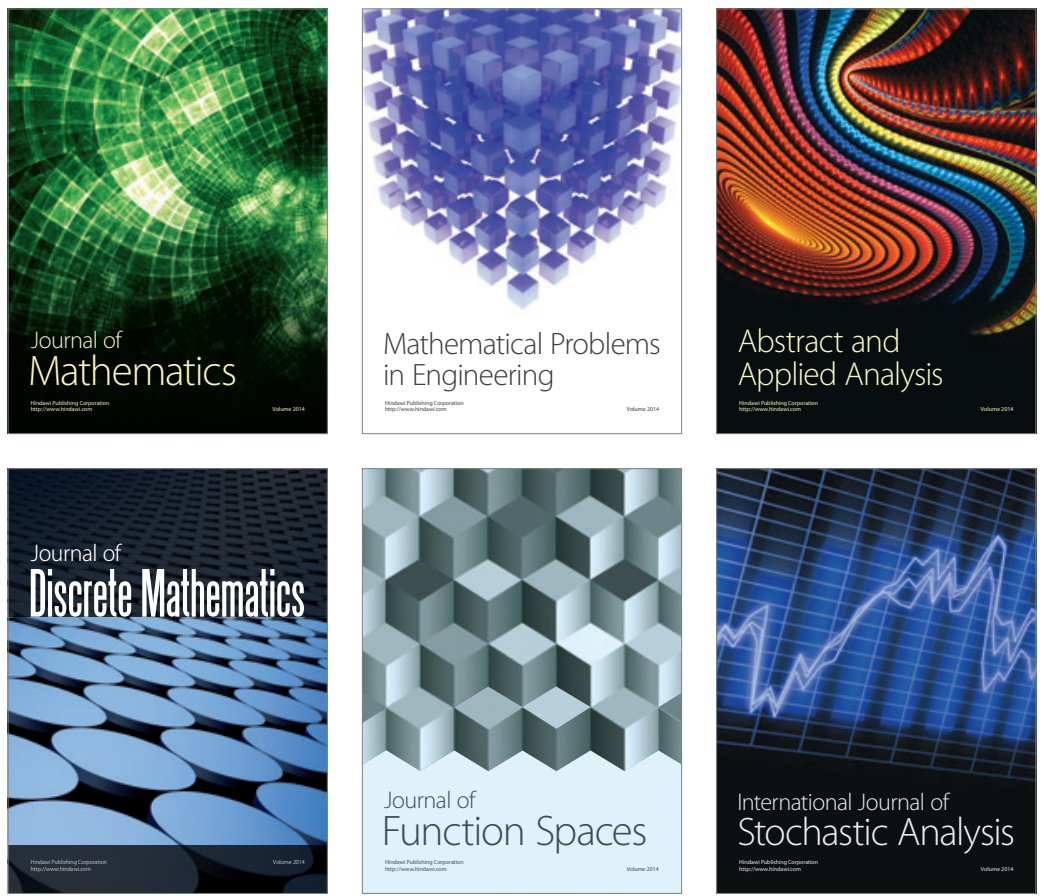

Journal of

Function Spaces

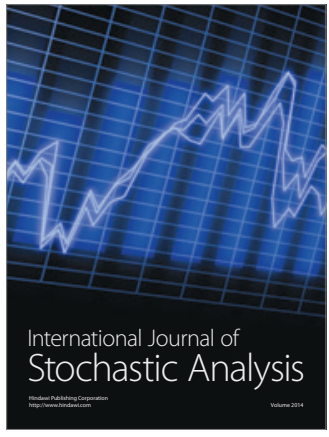

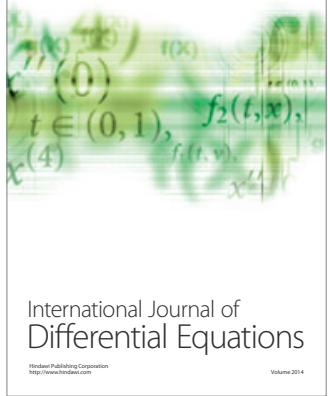
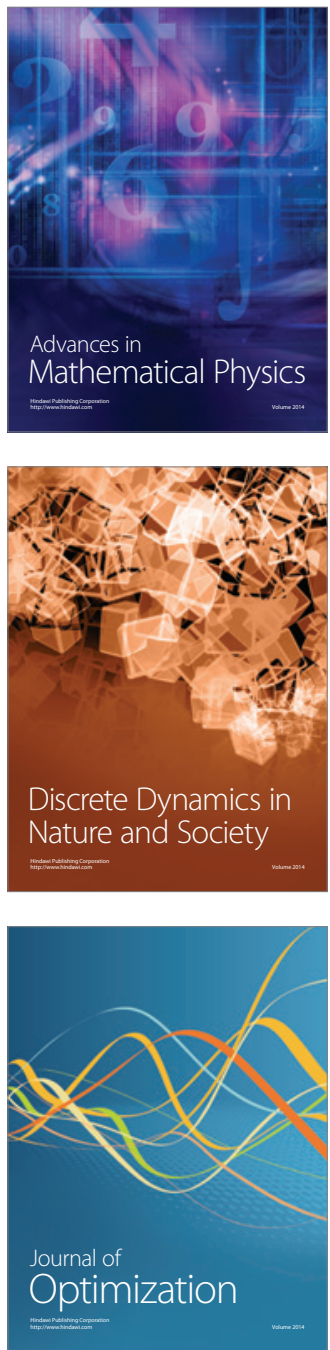